\title{
Hyperon electromagnetic timelike elastic form factors at large $q^{2}$
}

\author{
G. Ramalho $\odot,{ }^{1}$ M. T. Peña, ${ }^{2}$ and K. Tsushima $\oplus^{1}$ \\ ${ }^{1}$ Laboratório de Física Teórica e Computacional-LFTC, Universidade Cruzeiro do Sul and Universidade \\ Cidade de São Paulo, 01506-000, São Paulo, Sao Paulo, Brazil \\ ${ }^{2}$ Centro de Física Teórica e de Partículas (CFTP), Instituto Superior Técnico (IST), \\ Universidade de Lisboa, Avenida Rovisco Pais, 1049-001 Lisboa, Portugal
}

(Received 16 August 2019; published 21 January 2020)

\begin{abstract}
We present estimates of the hyperon elastic form factors for the baryon octet and the $\Omega^{-}$baryon for large four-momentum transfer squared $q^{2}$ in the timelike region $\left(q^{2}>0\right)$. Experimentally, those form factors can be extracted from the $e^{+} e^{-} \rightarrow B \bar{B}$ and $p \bar{p} \rightarrow B \bar{B}$ processes, where $B$ stands for a general baryon. Our results are based on calculations of the elastic electromagnetic form factors in the spacelike region $\left(Q^{2}=-q^{2}>0\right)$ within a covariant quark model. To connect the results in the spacelike region to those in the timelike region, we use asymptotic relations between the two regions which are constraints derived from analyticity and unitarity. We calculate the effective form factors $\left|G\left(q^{2}\right)\right|$ and compare them with the integrated cross section data $\sigma_{\mathrm{Born}}\left(q^{2}\right)$ from BABAR, BES III, and CLEO. The available data are at the moment restricted to $\Lambda, \Sigma^{0}, \Sigma^{-}, \Xi^{-}, \Xi^{0}$, and $\Omega^{-}$as well as to $e^{+} e^{-} \rightarrow \Lambda \bar{\Sigma}^{0}$ and $e^{+} e^{-} \rightarrow \Sigma^{0} \bar{\Lambda}$ reactions. Our results provide useful reference for future experiments and seem to indicate that the present data are still in the nonperturbative QCD region, while the onset for the asymptotic constraints from analyticity and unitarity happens much before the region of the perturbative QCD falloff of the form factors.
\end{abstract}

DOI: $10.1103 /$ PhysRevD.101.014014

\section{INTRODUCTION}

The understanding of internal structure of hadrons has been a great challenge after the discovery that the proton is not a pointlike particle. In the last decades, great progress has been made in the study of the nucleon electromagnetic structure, particularly through the scattering of electrons with nucleon targets $\left(\gamma^{*} N \rightarrow N\right.$ transition), which probes the spacelike momentum transfer kinematic region $\left(Q^{2} \geq 0\right)$ [1-4]. For hyperons $(B)$, however, it is difficult to get information on the internal structure based on the $\gamma^{*} B \rightarrow$ $B$ process due to their very short lifetimes. The available information is restricted at the moment only to the magnetic moments of a few hyperons (determined at $Q^{2}=0$ ).

The other possibility of disclosing the electromagnetic structure of baryons is $e^{+} e^{-}$scattering. It enables us to access the timelike region $\left(q^{2}=-Q^{2}>0\right)$ and was proposed a long time ago by Cabibbo and Gatto [5]; however, it became possible only recently. The $e^{+} e^{-} \rightarrow B \bar{B}$ (and the inverse) reactions open a new opportunity to study the role of valence quark effects, clusters of two-quark pairs (diquarks), and different quark compositions [6-12].

Published by the American Physical Society under the terms of the Creative Commons Attribution 4.0 International license. Further distribution of this work must maintain attribution to the author(s) and the published article's title, journal citation, and DOI. Funded by SCOAP .
The timelike region form factors appear as a viable tool to determine the hyperon structure, near the threshold as well as in the large- $q^{2}$ region, where in the latter perturbative effects are expected to dominate [4,5,11-16]. A significant amount of data are already available for the proton $\left(e^{+} e^{-} \rightarrow p \bar{p}\right)[4,13]$. In the present study, we focus on the reactions involving hyperons in the final states. Data associated with hyperon electromagnetic form factors in the timelike region also became available in facilities such as BABAR [17], BES-III [18,19], and CLEO [11,12]. The available data cover the high $-q^{2}$ region where we can expect to probe perturbative QCD (PQCD) physics.

From the theoretical side, there have been only a few different attempts in interpreting the hyperon timelike electromagnetic form factor data [20-27]. Although results from $e^{+} e^{-}$and $p \bar{p}$ annihilation experiments are already available or being planned in the near future, e.g., by the PANDA experiment at FAIR-GSI [28], theoretical calculations of hyperon electromagnetic timelike form factors are scarce. The results presented here intend to fill that gap.

In the large- $q^{2}$ region, one can expect the behavior predicted by PQCD [29-33]. However, some of the aspects from PQCD, including the $q^{2}$ dependence of the form factors, can be seen only at very high $q^{2}$. In the region covered by the present experiments, finite corrections for the large- $q^{2}$ behavior may be still relevant.

One of the goals of the present work is to provide calculations to be compared with the recent experimental 
determinations of the $e^{+} e^{-} \rightarrow B \bar{B}$ cross sections from CLEO, BABAR, and BES-III and to use them to guide new experiments also for larger $q^{2}$. The results presented here can be used to study the onset of the region for the validity of asymptotic behavior.

Our estimates are based on the results of a relativistic quark model for the spacelike region [34,35]. In this work, we focus on the general properties of the integrated cross section $\sigma_{\text {Born }}\left(q^{2}\right)$ and the effective form factor $\left|G\left(q^{2}\right)\right|$ for large $q^{2}$. Based on these, we test model-independent asymptotic relations between the form factors in the spacelike and the timelike regions [4]. We use those relations to calculate the magnetic and electric form factors in the timelike region and give estimates for the effective form factor $G\left(q^{2}\right)$ of the $\Lambda, \Sigma^{+}, \Sigma^{0}, \Sigma^{-}, \Xi^{0}, \Xi^{-}$, and $\Omega^{-}$ baryons. An interesting aspect that emerges from our results and the comparison with the data is that the region of $q^{2}$ where these model-independent relations may start to hold, differ from the (even larger) $q^{2}$ region of PQCD. This result is discussed and interpreted in terms of the physical scales included in our model.

In addition to the effective form factor $G\left(q^{2}\right)$, we calculate also the individual form factors $\left|G_{M}\right|$ and $\left|G_{E}\right|$ and determine their relative weights for the effective form factor. Most existing studies are based on the approximation $G_{M} \equiv G_{E}$ equivalent to $G\left(q^{2}\right)=G_{M}\left(q^{2}\right)$. However, it is important to notice that although by definition $G_{M}=G_{E}$ at the threshold of the timelike region $\left(q^{2}=4 M_{B}^{2}\right.$, where $M_{B}$ is the mass of the baryon), there is no proof that this relation holds for higher values of $q^{2}$. Therefore, in the present work we compare the result of the approximation $G=G_{M}$ with the exact result. The difference between the two results is a measure of the impact of $G_{E}$ in the magnitude of the effective form factor $G$.

It is worth mentioning that, at present, calculations of the timelike form factors based on a formulation in Minkowski space $\left(q^{2}=q_{0}^{2}-\mathbf{q}^{2}\right)$ are very important, since the timelike region, in practice, is still out of reach of the methods as lattice QCD simulations. Also, most of the DysonSchwinger-equation-based approaches formulated in the Euclidean space are still restricted to mass conditions compatible with singularity-free kinematic regions. Their extension to regions where singularities can be crossed requires elaborate contour deformation techniques [36].

This article is organized as follows: In the next section, we describe the general formalism associated with the $e^{+} e^{-} \rightarrow B \bar{B}$ processes and their relation with the form factors $G\left(q^{2}\right)$. In Sec. III, we review in detail the relativistic quark model used here, which was previously tested in calculations of several baryon elastic form factors in the spacelike region. The model-independent relations used for the calculations in the large- $q^{2}$ region are discussed in Sec. IV. The numerical results for the timelike form factors are presented and compared with the experimental data in
Sec. V. The outlook and conclusions are given in Sec. VI. Additional details are included in the Appendixes.

\section{FORMALISM}

We start our discussion with the formalism associated with spin- $1 / 2$ baryons with positive parity $\left(1 / 2^{+}\right)$. In the following, we represent the mass of the baryon by $M_{B}$ and use the notation $\tau=\frac{q^{2}}{4 M_{B}^{2}}$.

Within the one-photon-exchange approximation (equivalent to the impulse approximation in the spacelike region), one can interpret the $e^{+} e^{-} \rightarrow B \bar{B}$ reaction as the two-step process $e^{+} e^{-} \rightarrow \gamma^{*} \rightarrow B \bar{B}$, and the integrated cross section in the $e^{+} e^{-}$center-of-mass frame becomes $[12,13,37]$

$$
\sigma_{\mathrm{Born}}\left(q^{2}\right)=\frac{4 \pi \alpha^{2} \beta C}{3 q^{2}}\left(1+\frac{1}{2 \tau}\right)\left|G\left(q^{2}\right)\right|^{2},
$$

where $G\left(q^{2}\right)$ is an effective form factor for the baryon $B$ (spin-1/2 and positive parity), $\alpha \simeq 1 / 137$ is the finestructure constant, $\beta$ is a kinematic factor defined by $\beta=\sqrt{1-\frac{1}{\tau}}$, and $C$ is a factor which depends on the charge of the baryon. The factor $C$ is equal to 1 for neutral baryons. For charged baryons $C$, it takes into account the Coulomb effects near the threshold $[3,12,13,38]$ given by the Sommerfeld-Gamow factor $C=\frac{y}{1-\exp (-y)}$, with $y=\frac{\pi \alpha}{\beta} \frac{2 M_{B}}{\sqrt{q^{2}}}$. In the region of interest of the present study, at large $q^{2}(\tau \gg 1)$, one has $C \simeq 1$.

The magnitude of the effective form factor $G$ is defined by the combination of the electric and magnetic form factors $[12,13,37]$ as

$$
\begin{aligned}
\left|G\left(q^{2}\right)\right|^{2} & =\left(1+\frac{1}{2 \tau}\right)^{-1}\left[\left|G_{M}\left(q^{2}\right)\right|^{2}+\frac{1}{2 \tau}\left|G_{E}\left(q^{2}\right)\right|^{2}\right], \\
& =\frac{2 \tau\left|G_{M}\left(q^{2}\right)\right|^{2}+\left|G_{E}\left(q^{2}\right)\right|^{2}}{2 \tau+1} .
\end{aligned}
$$

Equations (2.1) and (2.2) are very useful, since they mean that one can describe the integrated cross section $\sigma_{\text {Born }}$ from the knowledge of a unique effective function $G\left(q^{2}\right)$ defined by the magnetic and the electric form factors. Note that the form factors $G_{M}$ and $G_{E}$ are complex functions of $q^{2}$ in the timelike region. The relations (2.1) and (2.2) are particularly practical to calculate $\sigma_{\text {Born }}\left(q^{2}\right)$, because they enable us to estimate the integrated cross section without taking into account the relative phases between the form factors $G_{M}$ and $G_{E}$.

Assuming charge invariance of the electromagnetic interaction, namely, that the spacelike and timelike photon-nucleon vertices $\gamma p p$ and $\gamma p \bar{p}$ are the same, we can estimate the timelike form factors in the timelike region from the form factors in the spacelike (SL) region $G_{M}^{\mathrm{SL}}\left(-q^{2}\right)$ and $G_{E}^{\mathrm{SL}}\left(-q^{2}\right)$ by applying the large- $\left|q^{2}\right|$ model-independent relations [4], 


$$
\begin{aligned}
& G_{M}\left(q^{2}\right) \simeq G_{M}^{\mathrm{SL}}\left(-q^{2}\right), \\
& G_{E}\left(q^{2}\right) \simeq G_{E}^{\mathrm{SL}}\left(-q^{2}\right),
\end{aligned}
$$

and therefore, restricting our results to the very-large- $q^{2}$ region, where the form factors are real functions to fulfill the Schwarz reflection principle. These asymptotic relations are a consequence of general physical and mathematical principles: unitarity as well as the Phragmén-Lindelöf theorem, which is valid for analytic functions (proved in Ref. [4]). They are exact in the mathematical $q^{2} \rightarrow \infty$ limit, and they imply that the imaginary part of the form factors in the timelike region goes to zero in that limit.

In the present work, we use a quark model developed in the spacelike region $[34,35]$ to estimate the magnetic and electric form factors in the timelike region based on Eqs. (2.3) and (2.4). The discussion on how these relations can be corrected for finite $q^{2}$ is made in Sec. IV. Deviations from those estimates may indicate that the imaginary parts of the form factors in the considered timelike region cannot be neglected.

We will investigate, by comparing with the data, the degree of validity of those relations for finite $q^{2}$. Increasing the value of $q^{2}$, we can tentatively look for the onset of the region where they may start to be a fairly good approximation. It turns out that this happens much below the region where the PQCD falloff of the form factors starts to emerge, as our results will show. We also provide estimates for $q^{2}>20 \mathrm{GeV}^{2}$ for comparison with future experiments.

The formalism used in the discussion of $1 / 2^{+}$baryons can also be extended to $3 / 2^{+}$baryons based on the effective form factor (2.2) reinterpreting $G_{M}$ as a combination of the magnetic dipole and magnetic octupole form factors, and $G_{E}$ as a combination of the electric charge and electric quadrupole form factors [20]. The expressions associated with $G_{M}$ and $G_{E}$ for $3 / 2^{+}$baryons are presented in Appendix A. Using those expressions, we calculate our results for the $\Omega^{-}$baryon.

Before presenting the results of the extension of our model to the timelike region, we present a review of the covariant spectator quark model in the spacelike regime that sustains the application here.

\section{COVARIANT SPECTATOR QUARK MODEL}

We restrict our study to baryons with one or more strange quarks (hyperons). In our estimates, we use the covariant spectator quark model. The covariant spectator quark model has been applied to the studies of the electromagnetic structure of several baryons, including nucleon, octet baryons, and decuplet baryons (including $\Omega^{-}$) in the spacelike region [34,35,39-53].

The model is based on three basic ingredients:

(1) The baryon wave function $\Psi_{B}$ rearranged as an active quark and a spectator quark pair is represented in terms of the spin-flavor structure of the individual quarks with $S U_{S}(2) \times S U_{F}(3)$ symmetry [35,39].

(2) By applying the impulse approximation, after integrating over the quark-pair degrees of freedom, the three-quark system transition matrix element can be reduced to that of a quark-diquark system parametrized by a radial wave function $\psi_{B}[35,39,40]$.

(3) The electromagnetic structure of the quark is parametrized by the quark form factors, $j_{1}$ (Dirac) and $j_{2}$ (Pauli) according to the flavor content, which encode the substructure associated with the gluons and quark-antiquark effects and are parametrized using the vector meson dominance (VMD) mechanism $[35,46,49]$.

Concerning the first two points above, the literature emphasizes the role of diquarks in the baryons [6-11]. Our model, although based on a quark-diquark configuration, cannot be interpreted as a quark-diquark model in the usual sense, i.e., a diquark as a pole of the quark-quark amplitude $[35,39,40]$. In our model, the internal quark-quark motion is integrated out at the level of impulse approximation, but its spin structure signature survives [39]. Therefore, the electromagnetic matrix element involves an effective quark-diquark vertex where the diquark is not pointlike [40].

Another difference between our model and the usual quark-diquark models is that we explicitly symmetrize in all quark pairs applying the $S U(3)$ flavor symmetry [34,35]. Since it is well known that the exact $S U(3)$ flavor symmetry models are expected to fail due to the mass difference between the light quarks ( $u$ and $d$ ) and the strange quarks, we break $S U$ (3) flavor symmetry in two levels. We break the symmetry at the level of the radial wave functions by using different forms for those functions for systems with a different number of strange quarks $\left(N_{s}=0,1,2\right.$ for the baryon octet and $N_{s}=0,1,2,3$ for the baryon decuplet) $[34,35]$. We break the $S U(3)$ flavor symmetry also at the level of the quark current by considering different $Q^{2}$ despondence for the different quark sectors (isoscalar, isovector, and strange quark components).

\section{A. Octet baryon wave functions}

The octet baryon wave functions associated with a quark-diquark system in the $S$-wave configuration can be expressed in the form [34,52]

$$
\Psi_{B}(P, k)=\frac{1}{\sqrt{2}}\left[\phi_{S}^{0}\left|M_{A}\right\rangle+\phi_{S}^{1}\left|M_{S}\right\rangle\right] \psi_{B}(P, k),
$$

where $P(k)$ are the baryon (diquark) momentum, $\phi_{S}^{0,1}$ are the spin wave functions associated with the components $S=0$ (scalar) and $S=1$ (vector) of the diquark states, and $\left|M_{A}\right\rangle$, and $\left|M_{S}\right\rangle$ are the mixed antisymmetric and mixed symmetric flavor states of the octet. The explicit expressions for $\left|M_{A}\right\rangle$ and $\left|M_{S}\right\rangle$ and for $\phi_{S}^{0,1}$ are included in Appendix B. For more details, see Refs. [34,39,52]. 
Since the baryons are on shell and the intermediate diquark in the covariant spectator model is taken also to be on shell, the radial wave functions $\psi_{B}$ can be written in a simple form using the dimensionless variable $\chi_{B}$ :

$$
\chi_{B}=\frac{\left(M_{B}-m_{D}\right)^{2}-(P-k)^{2}}{M_{B} m_{D}},
$$

where $m_{D}$ is the diquark mass [39]. One can now write the radial wave functions in the Hulthen form according to $[34,39]$

$$
\psi_{B}(P, k)=\frac{N_{B}}{m_{D}\left(\beta_{1}+\chi_{B}\right)\left(\beta_{i}+\chi_{B}\right)},
$$

where $N_{B}$ is a normalization constant, and $\beta_{i}(i=1,2,3,4)$ are momentum-range parameters (in units $M_{B} m_{D}$ ). The form of our baryon wave functions (3.3) was judiciously chosen to produce at large $Q^{2}$ the same behavior of the form factors as PQCD $[39,50]$ as discussed in Sec. III D.

In Eq. (3.3), $\beta_{1}$ is the parameter which establishes the long-range scale $\left(\beta_{1}<\beta_{2}, \beta_{3}, \beta_{4}\right)$ common to all the octet baryons, and $\beta_{i}(i=2,3,4)$ are parameters associated with the short-range scale, varying with the different quark flavor contents. The short-range scale is determined by $\beta_{2}(N), \beta_{3}(\Lambda$ and $\Sigma)$, and $\beta_{4}(\Xi)$.

The magnitudes of $\beta_{i}$ establish the shape of the radial wave function and determine the falloff of the baryon form factors. Heavier baryons have slower falloffs [34]. According to the uncertainty principle, the values of the parameters $\beta_{i}(i=2,3,4)$ can also be interpreted in terms of the compactification in space of the baryons. The relative ordering $\beta_{2}>\beta_{3}>\beta_{4}$ specifies that $\Lambda$ and $\Sigma^{0, \pm}$ are more compact than the nucleon, and that $\Xi^{0,-}$ are more compact than $\Lambda$ and $\Sigma^{0, \pm}$.

\section{B. Electromagnetic current}

The contribution of the valence quarks for the transition current in relativistic impulse approximation is expressed in terms of the quark-diquark wave functions $\Psi_{B}$ by $[34,39]$

$$
J_{B}^{\mu}=3 \sum_{\Gamma} \int_{k} \bar{\Psi}_{B}\left(P_{+}, k\right) j_{q}^{\mu} \Psi_{B}\left(P_{-}, k\right),
$$

where $j_{q}^{\mu}$ is the quark current operator, $P_{+}, P_{-}$, and $k$ are the final, initial, and diquark momenta, respectively, and $\Gamma$ labels the diquark scalar and vector diquark polarizations. The factor 3 takes into account the contributions associated with the different diquark pairs, and the integral symbol represents the covariant integration in the on-shell diquark momentum.
In Eq. (3.4), the quark current has a generic structure

$$
j_{q}^{\mu}=j_{1}\left(Q^{2}\right) \gamma^{\mu}+j_{2}\left(Q^{2}\right) \frac{i \sigma^{\mu \nu}}{2 M_{N}},
$$

where $M_{N}$ is the nucleon mass, and $j_{i}(i=1,2)$ are $S U(3)$ flavor operators.

The components of the quark current $j_{i}(i=1,2)$ can be decomposed as the sum of operators acting on the third quark in the $S U(3)$ flavor space

$j_{i}\left(Q^{2}\right)=\frac{1}{6} f_{i+}\left(Q^{2}\right) \lambda_{0}+\frac{1}{2} f_{i-}\left(Q^{2}\right) \lambda_{3}+\frac{1}{6} f_{i 0}\left(Q^{2}\right) \lambda_{s}$,

where

$\lambda_{0}=\left(\begin{array}{lll}1 & 0 & 0 \\ 0 & 1 & 0 \\ 0 & 0 & 0\end{array}\right), \quad \lambda_{3}=\left(\begin{array}{ccc}1 & 0 & 0 \\ 0 & -1 & 0 \\ 0 & 0 & 0\end{array}\right)$,

$\lambda_{s} \equiv\left(\begin{array}{ccc}0 & 0 & 0 \\ 0 & 0 & 0 \\ 0 & 0 & -2\end{array}\right)$

are the flavor operators. These operators act on the quark wave function in flavor space $q=(u d s)^{T}$. The functions $f_{i+}, f_{i-}(i=1,2)$ represent the quark isoscalar and isovector form factors, respectively, based on the combinations of the quarks $u$ and $d$. The functions $f_{i 0}(i=1,2)$ represent the structure associated with the strange quark. The explicit form for the quark form factors is included in Appendix B.

For the discussion of the results of this paper, it is relevant that the parametrization of these form factors is based on the VMD picture. The dressed photon-quark coupling is tied to the vector meson spectra. Therefore, the isoscalar and the isovector form factors include contributions from the $\rho$ and $\omega$ mass poles in the light quark sector. As for the strange quark form factors, we include a dependence on the $\phi$ mass pole. In both cases, we include also a contribution of an effective heavy meson with mass $2 M_{N}$ in order to take into account shorter-range effects in the quark current. The parametrization of the current for the three quark sectors includes five parameters (coefficients of the vector meson terms) in addition to the three quark anomalous magnetic moments.

\section{Model for the nucleon and decuplet baryons}

The model was first applied to the study of the electromagnetic structure of the nucleon. The free parameters of the model (in the quark current and in the radial wave functions) were calibrated by the electromagnetic form factor data for the proton and the neutron [39]. The nucleon data are well described without an explicit inclusion of pion cloud contributions. 
Taking advantage of the VMD form of the quark current and of the covariant form of the radial wave function, the model was extended to the lattice QCD regime $[35,46,49]$. This extension was performed by replacing the vector meson and nucleon masses in the VMD parametrization of the current and in the baryon wave functions by the nucleon and vector meson masses from the lattice. This extension is valid for the region of the large pion masses, where there is a suppression of the meson cloud effects.

The extension has proved to be very successful in the description of the lattice QCD data for the nucleon and $\gamma^{*} N \rightarrow \Delta(1232)$ transition for pion masses above $400 \mathrm{MeV}$ [46,49]. In the case of the $\gamma^{*} N \rightarrow \Delta(1232)$ transition, the lattice data enabled us to fix the valence quark contribution, and after the extrapolation to the physical pion mass limit, indirectly infer from the physical data the meson cloud effects $[46,54]$. The meson cloud effects were then seen to be significant in the case of the $\Delta(1232)$ due to the vicinity of its mass to the pion-nucleon threshold [45-47].

The formalism was later applied to all baryons of the decuplet using an $S U_{F}(3)$ extension of the model for the $\Delta(1232)[45,46,51]$ constrained by the scarce available lattice data for the decuplet baryon electromagnetic form factors and the experimental magnetic moment of the $\Omega^{-}$ [35]. The strange quark component of the current and the decuplet radial wave functions were then determined by the fit to the data (lattice QCD and experimental $\Omega^{-}$magnetic moment). No meson cloud contributions were considered in this description of the baryon decuplet since those effects are suppressed in lattice calculations. Also, the only physical information on the meson cloud comes from the $\Delta(1232)$ calibrated in the previous works [46], and for the $\Omega^{-}$. In this last case, meson cloud effects are expected to be very small, since pion excitations are suppressed due to the content of the valence quark core (only strange quarks) implying reduced kaon excitations given the large mass of the kaon $[35,48]$.

The model for the $\Omega^{-}$was later recalibrated with the first lattice QCD calculation of the $\Omega^{-}$form factors at the physical mass point, which we used to determine the electric quadrupole and magnetic octupole moments [48].

\section{Model for the octet baryons}

Using the $S U(3)$ quark current determined in the studies of the nucleon and decuplet baryon systems, the covariant spectator quark model was also extended to the octet baryon systems. However, different from the decuplet case, where a fair description of the data can be obtained based exclusively on the valence quark degrees of freedom, in the case of the octet, there is evidence that the pion cloud effects are significant [52]. Therefore, in the model for the baryon octet, in addition to the valence quarks, we consider also explicit pion cloud contributions based on the $S U(3)$ pion-baryon interaction [34,52].
The valence quark contributions regulated by the radial wave functions (3.3) were fixed by lattice QCD data. The pion cloud contributions were calibrated by the physical data (nucleon electromagnetic form factors and octet magnetic moments). Compared to the previous studies of the nucleon [39], we readjusted the values of the momentum-range parameters $\beta_{1}$ and $\beta_{2}$ of the radial wave functions (3.3) and the quark anomalous moments $\kappa_{u}$ and $\kappa_{d}$ in order to take into account the effects of the pion cloud. More details can be found in Appendixes B 3 and B 4.

We discuss now the contributions from the valence quarks to the form factors. From the structure for the quark current and radial wave functions, we obtain the following expressions for the valence quark contributions to the octet baryon form factors:

$$
\begin{aligned}
F_{1 B}\left(Q^{2}\right)= & B\left(Q^{2}\right) \\
& \times\left[\frac{3}{2} j_{1}^{A}+\frac{1}{2} \frac{3-\tau}{1+\tau} j_{1}^{S}-2 \frac{\tau}{1+\tau} \frac{M_{B}}{M_{N}} j_{2}^{S}\right], \\
F_{2 B}\left(Q^{2}\right)= & B\left(Q^{2}\right) \\
\times & {\left[\left(\frac{3}{2} j_{2}^{A}-\frac{1}{2} \frac{1-3 \tau}{1+\tau} j_{2}^{S}\right) \frac{M_{B}}{M_{N}}-2 \frac{1}{1+\tau} j_{1}^{S}\right], }
\end{aligned}
$$

with $\tau=\frac{Q^{2}}{4 M_{B}^{2}}$, and

$$
B\left(Q^{2}\right)=\int_{k} \psi_{B}\left(P_{+}, k\right) \psi_{B}\left(P_{-}, k\right),
$$

the overlap integral between the initial and final scalar wave functions. The function $B\left(Q^{2}\right)$ is independent of the diquark mass [39].

The coefficients $j_{i}^{A, S}(i=1,2)$ are combinations of the quark form factors dependent on the baryon quark content. The explicit expressions are included in Appendix B. One concludes that the results are an interplay of both the structure of the quark form factors and of the radial wave functions.

The results for the electric and magnetic form factors are then determined by

$$
G_{E B}=F_{1 B}-\tau F_{2 B}, \quad G_{M B}=F_{1 B}+F_{2 B} .
$$

The asymptotic behavior of the form factors $G_{E}$ and $G_{M}$ is determined by the asymptotic results for $F_{1 B}$ and $F_{2 B}$ from Eqs. (3.8) and (3.9). The terms between brackets depend only on the quark form factors and for large $Q^{2}$, and contribute to $F_{1 B}$ and $Q^{2} F_{2 B}$ with constants. As a consequence, the results for $G_{E}$ and $G_{M}$ are determined at very large $Q^{2}$ by the function $B\left(Q^{2}\right)$, which in turn exclusively depends on the radial wave functions and their overlap. In Ref. [50], it was shown that if we use the radial wave functions (3.3), one has $B \propto 1 / Q^{4}$ plus logarithmic 
corrections. We can conclude then that the combination of the quark current with the radial structure induces falloffs for the form factors consistent with the power law of the PQCD result: $G_{E} \propto 1 / Q^{4}$ and $G_{M} \propto 1 / Q^{4}$, in addition to logarithmic corrections [30,31,33,50].

The deviations of our results from the simple power law $1 / Q^{4}$ are originated by contaminations from logarithmic corrections or from the difference of the quark form factors from their asymptotic result $\left(j_{1}^{A, S}, Q^{2} j_{2}^{A, S} \rightarrow\right.$ constant). The latter is regulated by large momentum scales associated with the VMD parametrization. But the different momentum falloff tails of the baryon form factors also play a role and relate to the difference in the flavor content of the constituent valence quarks described by the wave functions, as well as the VMD structure of the quark form factors.

As mentioned above, an accurate description of the electromagnetic structure of the octet baryons is achieved when we include an explicit parametrization of the pion cloud contributions [34,44]. The consequence of the introduction of the pion cloud effects is that the transition form factors (3.8) and (3.9) have additional contributions, which can be significant below $Q^{2}<2 \mathrm{GeV}^{2}$, and that the two contributions have to be normalized by a global factor $Z_{B}<1\left(\sqrt{Z_{B}}\right.$ is the factor associated with each wave function).

In the large- $Q^{2}$ region, the pion cloud contributions are suppressed, and the form factors are then reduced to

$$
G_{E B} \rightarrow Z_{B} G_{E B}, \quad G_{M B} \rightarrow Z_{B} G_{M B}
$$

where $G_{E B}$ and $G_{M B}$ on the rhs represent the valence quark estimate. From Eq. (3.12), we conclude that the pion cloud dressing affects only the normalization of the form factors at large $Q^{2}$. The normalization factor $Z_{B}$ depends only on a parameter associated with the pion cloud parametrization: the parameter which determines the pion cloud contribution to the proton charge $\left(Z_{N}\right)$. In Appendix B, we show that all normalization factors can be determined by the normalization of the nucleon wave functions $Z_{N}$. The values of $Z_{B}$ (between 0.9 and 1) are also presented in Appendix B.

Our calculations for the baryon octet presented in the next sections for the $Q^{2}>5 \mathrm{GeV}^{2}$ region depend essentially on seven parameters: four momentum-range parameters $\left(\beta_{i}\right)$, two anomalous magnetic moments $\left(\kappa_{u}\right.$ and $\left.\kappa_{d}\right)$, and one pion cloud parameter associated with the normalization of the octet baryon wave functions. The parametrization for the quark current was determined previously in the studies of the nucleon and baryon decuplet systems.

\section{MODEL-INDEPENDENT RELATIONS IN THE LARGE- $\boldsymbol{q}^{2}$ REGIME}

In the present work, we test the results of extrapolating the parametrizations in the spacelike region $\left(q^{2}=-Q^{2}<0\right)$ to the timelike region $\left(q^{2}>0\right)$. The calculation in the timelike

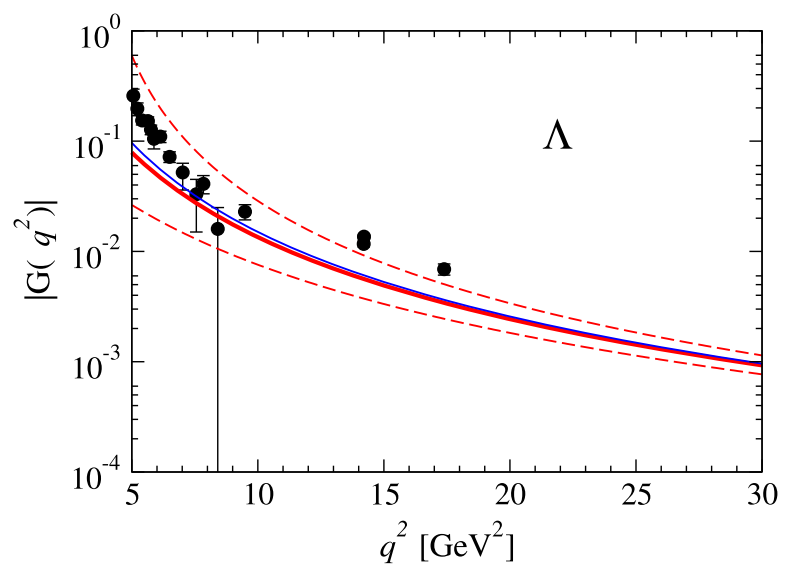

FIG. 1. Timelike form factor $G$ for the $\Lambda$. Data are from Refs. $[11,12,17,19]$. The thick solid line is based on Eqs. (4.1) and (4.2). The dashed lines represent the upper limit $G_{l}\left(q^{2}\right)=$ $G_{l}^{\mathrm{SL}}\left(4 M_{B}^{2}-q^{2}\right)$ and the lower limit $G_{l}\left(q^{2}\right)=G_{l}^{\mathrm{SL}}\left(-q^{2}\right)(l=M$, $E)$. The thin solid line is obtained with the approximation $G_{E}=G_{M}$.

region is based also on the model-independent relations (2.3) and (2.4) results for large $Q^{2}$.

Concerning the relations (2.3) and (2.4), they map the region of $\left.\left.q^{2}:\right]-\infty, 0\right]$ into the region $[0,+\infty$ [. Note, however, that $q^{2}=0$ is not the center point of the reflection symmetry that relates timelike and spacelike regions because of the unphysical gap region $] 0,4 M_{B}^{2}$ [ between them. The reflection symmetry center point between the two regions lies, in fact, inside this interval and can be tentatively taken as $q^{2}=2 M_{B}^{2}$ instead of $q^{2}=0$. This consideration leads us to correct the relations (2.3) and (2.4) by introducing finite corrections to $q^{2}$,

$$
\begin{aligned}
& G_{M}\left(q^{2}\right) \simeq G_{M}^{\mathrm{SL}}\left(2 M_{B}^{2}-q^{2}\right), \\
& G_{E}\left(q^{2}\right) \simeq G_{E}^{\mathrm{SL}}\left(2 M_{B}^{2}-q^{2}\right) .
\end{aligned}
$$

While the difference between using (2.3) and (2.4) and (4.1) and (4.2) is naturally negligible for very large $q^{2}$ and is immaterial in the mathematical $q^{2} \rightarrow \infty$ limit, it can be non-negligible otherwise. In the next section, we check that this is indeed the case when one gets to values in the range $q^{2}=10-20 \mathrm{GeV}^{2}$.

In the calculations presented in the next section, Eqs. (4.1) and (4.2) provide a central value for our results of the form factors, Eqs. (2.3) and (2.4), a lower limit, while the estimate where we replace $G_{l}^{\mathrm{SL}}\left(-q^{2}\right)$ by $G_{l}^{\mathrm{SL}}\left(4 M_{B}^{2}-q^{2}\right)(l=M, E)$ gives the upper limit.

An important point that is addressed in the next section is to know how far the region of the asymptotic relations (4.1) and (4.2) is from the PQCD region characterized by the relations $G_{M} \propto 1 / q^{4}$ and $G_{E} \propto 1 / q^{4}[30,31,33]$. 

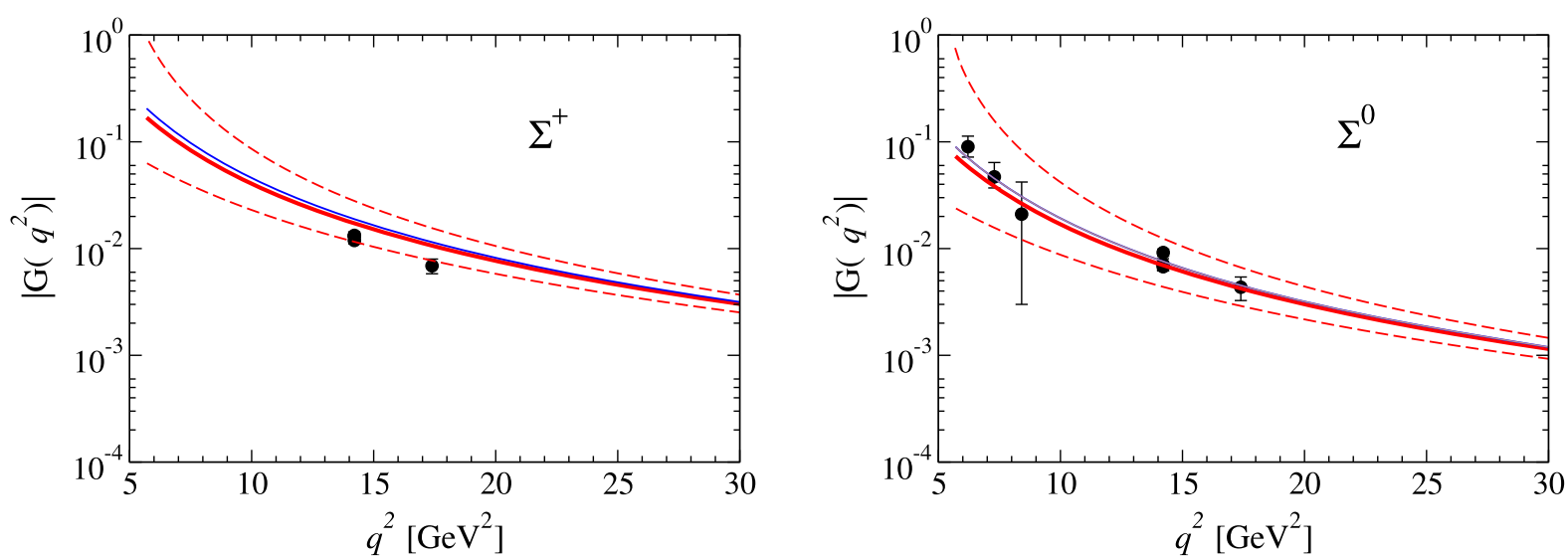

FIG. 2. Timelike form factor $G$ for the $\Sigma^{+}$(left) and $\Sigma^{0}$ (right). Data are from Refs. $[11,12,17,18]$. See also caption of Fig. 1 .
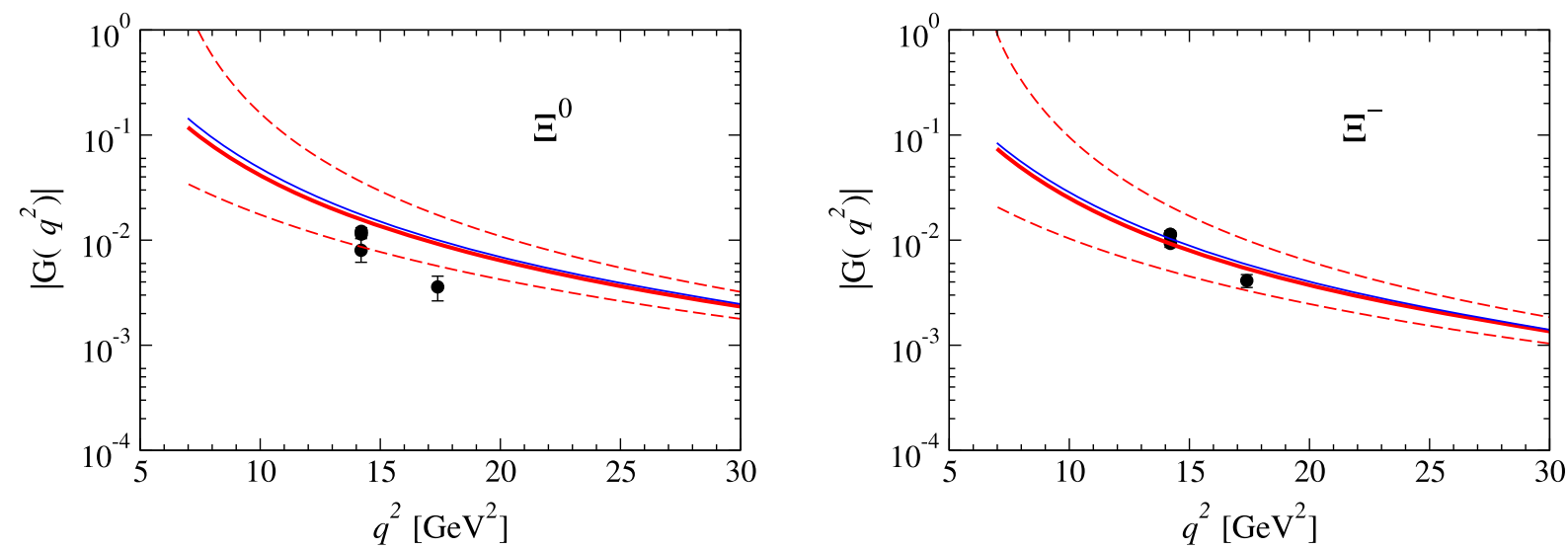

FIG. 3. Timelike form factor $G$ for the $\Xi^{0}$ (left) and $\Xi^{-}$(right). Data are from Refs. [11,12,18]. See also caption of Fig. 1.

\section{RESULTS}

In this section we present the results in the timelike region for the $\Lambda, \Sigma^{-}, \Sigma^{0}, \Xi^{-}$, and $\Xi^{0}$ of the baryon octet and also for the $\Omega^{-}$(baryon decuplet). The results for the baryon octet are based on the model from Ref. [34]. The results for the $\Omega^{-}$are based on the model from Ref. [48].

\section{A. Octet baryons}

The results of our model in the timelike region are presented in Figs. 1-3 for the cases of $\Lambda, \Sigma^{-}, \Sigma^{0}, \Xi^{-}$, and $\Xi^{0}$. The thick solid lines represent our best estimate based on Eqs. (4.1) and (4.2). The dashed lines represent the upper limit $G_{l}\left(q^{2}\right)=G_{l}^{\mathrm{SL}}\left(4 M_{B}^{2}-q^{2}\right)$ and the lower limit $G_{l}\left(q^{2}\right)=G_{l}^{\mathrm{SL}}\left(-q^{2}\right)(l=M, E)$. The thin solid line results are those obtained with the approximation $G_{E}=G_{M}$ and will be discussed later. Naturally, all curves get closer together as $q^{2}$ increases. In all cases, we use the experimental masses or the averages (respectively, for $\Sigma$ and $\Xi$ ). We recall that in the present model, the $S U(3)$ flavor symmetry is broken by the radial wave functions and that the quark electromagnetic structure is parametrized based on a VMD representation.

Our estimates are compared with the world data for the hyperon electromagnetic form factors in the timelike region. The data for the $\Lambda, \Sigma^{0}$, and $\Lambda \bar{\Sigma}^{0}$ (from $e^{+} e^{-} \rightarrow$ $\Lambda \bar{\Sigma}^{0}$ and $e^{+} e^{-} \rightarrow \Sigma^{0} \bar{\Lambda}$ reactions) for values of $q^{2}$ up to $9 \mathrm{GeV}^{2}$ are from BABAR [17]. There are also data from BESIII for the $\Lambda$ [19] below $q^{2}=10 \mathrm{GeV}^{2}$ and for $\Sigma^{0}, \Sigma^{+}, \Xi^{-}$, and $\Xi^{0}$ for $q^{2} \simeq 14.2 \mathrm{GeV}^{2}$ [ $\psi(3770)$ decay] [18]. Finally, there are data from CESR (CLEO-c detector) $[11,12]$ for the baryon octet $\left(\Lambda, \Lambda \bar{\Sigma}^{0}, \Sigma^{0}, \Sigma^{+}, \Xi^{-}\right.$, and $\left.\Xi^{0}\right)$ and $\Omega^{-}$for $q^{2} \simeq$ 14.2 and $17.4 \mathrm{GeV}^{2}[\psi(3770)$ and $\psi(4170)$ decays]. In the near future, we expect results on the proton-antiproton scattering from PANDA $(p \bar{p} \rightarrow B \bar{B})$ [28].

Contrary to the case of the proton form factor data in the timelike region, which is about 2 times larger than those in the spacelike region [6,55-57], the hyperon form factors have about the same magnitude (central value lines in the figures) in both regions (spacelike and timelike). 
TABLE I. Comparison between the ratios between the experimental value $\left(G^{\exp }\right)$ and the model estimate of $G\left(G^{\bmod }\right)$ for the different baryons, for $q^{2} \simeq 14.2$ and $17.4 \mathrm{GeV}^{2}[11,12]$. The last line indicates the average of all baryons.

\begin{tabular}{cc}
\hline \hline$B$ & $\left\langle\frac{G^{\text {exp }}}{G^{\mathrm{mod}}}\right\rangle$ \\
\hline$\Lambda$ & 2.19 \\
$\Sigma^{+}$ & 0.65 \\
$\Sigma^{0}$ & 1.08 \\
$\Xi^{-}$ & 1.08 \\
$\Xi^{0}$ & 0.60 \\
Average & 1.12 \\
\hline \hline
\end{tabular}

Our results suggest that the available data may already be within the asymptotic region where Eqs. (4.1) and (4.2) are valid, with the deviations consistent with a variation of the argument of $G$ from $q^{2}$ (lower limit) up to $q^{2}-4 M_{B}^{2}$ (upper limit), denoting that the reflection center point is within the unphysical region. In the model of Ref. [58], this seems also to be the case.

From the figures, we can conclude that our estimates (central values) are close to the data for $q^{2}>8 \mathrm{GeV}^{2}$ in most cases. For the $\Lambda$ case, our results underestimate the data. For the $\Sigma^{+}$and $\Xi^{0}$ cases, our results overestimate the data. However, in general, our results are reasonably close to the data. To compare our results with the data for larger values of $q^{2}\left(q^{2} \simeq 14.2\right.$ and $17.4 \mathrm{GeV}^{2}$ from CLEO-c $[11,12])$, we show in Table I the average ratios of the experimental values and our estimates. Note that for the $\Xi^{0}$, we have an underestimate of $40 \%(\approx 0.6)$, and for the $\Lambda$, an overestimate of more than $100 \%(\approx 2.2)$. This feature is similar to the proton case. When we average in the baryon indices, however, we obtain a ratio of 1.12, meaning that the baryon average value is very close to our model estimate.

Although our results corroborate the idea that the region shown is close to the region where the relations (4.1) and (4.2) hold, that does not mean that the region is close to the PQCD region, where $G_{E} \propto 1 / q^{4}$ and $G_{M} \propto 1 / q^{4}$. Calculations in the spacelike region where we consider the leading order term of the asymptotic quark current suggest that the first signs of the PQCD behavior $G_{E} \propto$ $1 / Q^{4}$ and $G_{M} \propto 1 / Q^{4}$ (with log corrections) appear only for $q^{2} \approx 100 \mathrm{GeV}^{2}$. An example of the convergence for $\left|G_{M}\right|$ and $\left|G_{E}\right|$ to the perturbative regime is presented in Fig. 4 for the case of $\Sigma^{+}$. The lines with the label "Model" indicate the exact result; the lines with the label "Large $Q^{2}$ " indicate the calculation with the asymptotic quark current. Similar behavior can be observed for the other hyperons.

The sharp minimum on $\left|G_{E}\right|$ is a consequence of the zero crossing for $Q^{2} \simeq 10 \mathrm{GeV}^{2}\left(G_{E}\right.$ becomes negative above that point). This case is similar to the case of the proton, where there is the possibility of a zero crossing near $Q^{2} \simeq$ $9 \mathrm{GeV}^{2}$ according to recent measurements at Jefferson Lab [59]. The zero crossing is also expected for other hyperons.

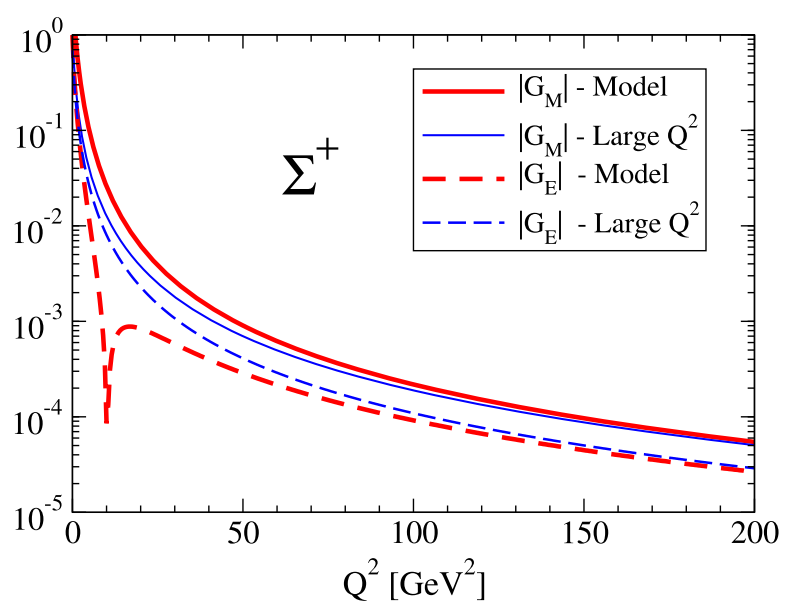

FIG. 4. $\Sigma^{+}$form factors. Comparison between the results for $\left|G_{M}\right|$ and $\left|G_{E}\right|$ for a model with an exact quark current (Model) and the results where we consider only the leading order term in $Q^{2}$ for the quark current (Large $Q^{2}$ ).

That the leading order PQCD behavior of the form factors only appears for higher $q^{2}$ can be interpreted as the interplay between the meson masses that enter the model through the constituent quark electromagnetic form factors (describing the photon-quark coupling) and the tail of the baryon wave functions that enter the overlap integral. On one hand, the quark electromagnetic form factors carry information on the meson spectrum being parametrized using the VMD mechanism in our model. Depending on the hyperon flavor, one has different contributions from the poles associated with light vector mesons $(0.8-1.0 \mathrm{GeV})$ and an effective heavy vector meson $(1.9 \mathrm{GeV})$. Those vector meson masses provide a natural scale, which regulates the falloff of the hyperon electromagnetic form factors. Note that the light vector meson masses (0.8$1.0 \mathrm{GeV}$ ) correspond to a large scale compared to the low$Q^{2}$ scale of QCD $(\sim 0.3 \mathrm{GeV})$ and of the constituent quark masses. On the other hand, the momentum falloff tail of the wave functions of the heavy baryons is associated with larger cutoffs. The same effect is observed in lattice QCD simulations, where form factors associated with larger pion masses exhibit slower falloffs in $Q^{2}[46,49,60]$.

For a detailed comparison with the present and future data, we present in Tables II and III our estimates for $G$ at larger values of $q^{2}$. Note in particular that we present predictions for $\Sigma^{-}$, a baryon for which there are no data at the moment. The results in the tables can be used to calculate the ratios between the form factors associated with different baryons.

From the previous analysis, we can conclude that the effective form factor $G$ for most of the octet baryons with strange quarks (hyperons) is well described by our approximated $S U_{F}(3)$ model combined with the asymptotic relations (4.1) and (4.2), since the data lie within the upper and lower limits of the theoretical uncertainty. 
TABLE II. Estimates for the $\Sigma$ effective form factor $G$ in units $10^{-3}$.

\begin{tabular}{lccc}
\hline \hline$q^{2}\left(\mathrm{GeV}^{2}\right)$ & $\Sigma^{+}$ & $\Sigma^{0}$ & $\Sigma^{-}$ \\
\hline 10 & 40.5 & 16.8 & 10.7 \\
15 & 15.1 & 6.09 & 4.12 \\
20 & 7.68 & 3.01 & 2.19 \\
25 & 4.58 & 1.76 & 1.36 \\
30 & 3.03 & 1.15 & 0.923 \\
35 & 2.14 & 0.803 & 0.667 \\
40 & 1.60 & 0.592 & 0.503 \\
45 & 1.24 & 0.453 & 0.393 \\
50 & 0.980 & 0.358 & 0.315 \\
55 & 0.799 & 0.290 & 0.260 \\
60 & 0.663 & 0.239 & 0.216 \\
\hline \hline
\end{tabular}

TABLE III. Estimates for the $\Lambda$ and $\Xi$ effective form factor $G$ in units $10^{-3}$.

\begin{tabular}{lccc}
\hline \hline$q^{2}\left(\mathrm{GeV}^{2}\right)$ & $\Lambda$ & $\Xi^{0}$ & $\Xi^{-}$ \\
\hline 10 & 13.4 & 41.4 & 24.9 \\
15 & 4.90 & 13.6 & 7.99 \\
20 & 2.43 & 6.41 & 3.75 \\
25 & 1.43 & 3.65 & 2.13 \\
30 & 0.927 & 2.33 & 1.36 \\
35 & 0.648 & 1.61 & 0.933 \\
40 & 0.476 & 1.17 & 0.679 \\
45 & 0.365 & 0.893 & 0.514 \\
50 & 0.288 & 0.700 & 0.402 \\
55 & 0.233 & 0.564 & 0.323 \\
60 & 0.192 & 0.463 & 0.264 \\
\hline \hline
\end{tabular}

\section{Discussion}

In the literature, there are a few estimates of hyperon form factors based on vector meson dominance [20,22]. The first calculation (1977) [20] was performed with no adjustable parameters, before the first measurements (Orsay, 1990) [61]. Those estimates differ from the recent measurements by an order of magnitude [11,12]. An improved VMD estimate (1993) [22] gave results closer to the $\Lambda$ data under the condition $G_{M}=G_{E}[11,14]$. There are also recent estimates for the $\Lambda$ and $\Sigma^{0}$ form factors based on phenomenological parametrizations of the baryon-antibaryon interaction [23], asymptotic parametrizations, and vector meson dominance parametrizations of the form factors [25-27].

In our model, the $S U_{F}(2)$ symmetry is broken at the quark level since we use different parametrizations for the isoscalar and isovector quark form factors. The dependence on the isovector component is more relevant for the case of the neutron for which there are almost no data available $[3,62,63]$, and for the $e^{+} e^{-} \rightarrow \Lambda \bar{\Sigma}^{0}$ and $e^{+} e^{-} \rightarrow \Sigma^{0} \bar{\Lambda}$ reactions, which we discuss at the end of the present section.

We now discuss the difference in magnitude between the electric and magnetic form factors of the octet baryon members. The absolute value of the magnetic form factor $\left|G_{M}\right|$ is represented in Figs. 1-3 by the thin solid line, which is, with no exception, just a bit above the central (thick solid line). Those results mean that the magnetic form factor is larger than the electric form factor $\left(\left|G_{E}\right|<\left|G_{M}\right|\right)$ for $\Lambda, \Sigma^{+}, \Sigma^{0}, \Xi^{0}$, and $\Xi^{-}$. This conclusion is a consequence of the definition of $\left|G\left(q^{2}\right)\right|^{2}$ given by Eq. (2.2). If we express $\left|G_{E}\right|$ in terms of the ratio $\alpha_{G}=\frac{\left|G_{E}\right|}{\left|G_{M}\right|}$, we obtain $|G|^{2}=\left|G_{M}^{2}\right|\left(1+\frac{\alpha_{G}^{2}-1}{1+2 \tau}\right)$. Since the thick solid line is the result for the full $\left|G\left(q^{2}\right)\right|$ function, and the thin solid line is the result from assuming $\left|G\left(q^{2}\right)\right|=\left|G_{M}\left(q^{2}\right)\right|$, we conclude that although $\left|G_{E}\right|<\left|G_{M}\right|$, the two form factors have similar magnitudes.

Our model can also be applied for the $\Lambda \bar{\Sigma}^{0}$ and $\bar{\Lambda} \Sigma^{0}$ form factors ( $e^{+} e^{-} \rightarrow \Lambda \bar{\Sigma}^{0}$ and $e^{+} e^{-} \rightarrow \bar{\Lambda} \Sigma^{0}$ reactions). It is important to notice, however, that the analysis of the $e^{+} e^{-} \rightarrow \Lambda \bar{\Sigma}^{0}$ and the $e^{+} e^{-} \rightarrow \bar{\Lambda} \Sigma^{0}$ reactions is a bit more intricate than the analysis for the $e^{+} e^{-} \rightarrow B \bar{B}$ reactions associated with the elastic form factors. In this case, there are two possible final states $\left(\Lambda \bar{\Sigma}^{0}\right.$ and $\left.\bar{\Lambda} \Sigma^{0}\right)$. From the experimental point of view, this implies that the background subtraction in the cross section analysis is also more complex due to the proliferation of decay channels, including the $\Lambda$ and $\Sigma^{0}$ decays and the decays of the corresponding antistates.

From the theoretical point of view, the $\gamma^{*} \Lambda \rightarrow \Sigma^{0}$ transition form factors in the spacelike region are difficult to test due to the lack of experimental data: There are no experimental constraints for the electric and magnetic form factors, except for the transition magnetic moment. We do not discuss here in detail our results for the $\gamma^{*} \Lambda \rightarrow \Sigma^{0}$ transition form factors due to the experimental ambiguities and also because the main focus of this work is the octet baryon electromagnetic form factors. Still, we mention that we predict the dominance of the meson cloud contributions for $G_{E}$ and of the valence quark contributions for $G_{M}$ [44]. At large $Q^{2}$, the magnetic form factor dominates over the electric form factor. This dominance is then mirrored to the timelike region. Our estimate of $G$ in the timelike region overestimates the data by about an order of magnitude, suggesting that the magnetic form factor dominance is not so strong in the timelike region. Another interesting theoretical aspect related to the $\gamma^{*} \Lambda \rightarrow \Sigma^{0}$ transition is its isovector character. This property can be studied in the near future once accurate timelike data for the neutron become available at large $q^{2}$. From the combination of proton and neutron data, we can determine the isovector component of the nucleon form factors. Then, those can be used to study the $\gamma^{*} \Lambda \rightarrow \Sigma^{0}$ transition form factors.

\section{B. $\boldsymbol{\Omega}^{-}$form factors}

CLOE-c provided the first measurements of the $\Omega^{-}$form factors for nonzero $q^{2}[11,12]$. Our results for the $\Omega^{-}$form 

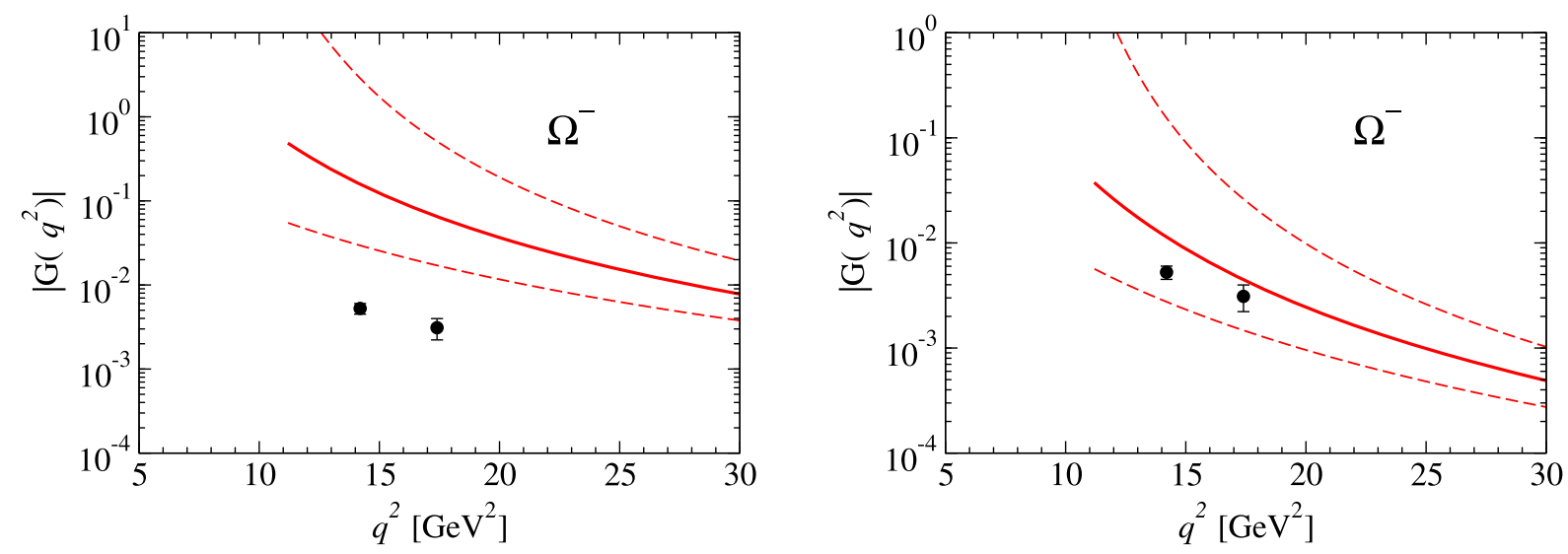

FIG. 5. Timelike form factor $G$ for the $\Omega^{-}$. We present the full result in the left panel (including $G_{E 2}$ and $G_{M 3}$ ). In the right panel, we drop the higher order multipoles $\left(G_{E 2}\right.$ and $\left.G_{M 3}\right)$. The model from Ref. [48] predicts a large magnitude for $G_{M 3}$. The timelike data support the estimates with a much smaller $G_{M 3}$. Data are from Ref. [11]. See also caption of Fig. 1.

factors are very important, because theoretical studies of the $\Omega^{-}$are scarce due to its unstable character. Fortunately, for the $\Omega^{-}$, lattice QCD simulations at the physical point (i.e., physical strange quark mass) exist [64]. Since those simulations are at the physical point and the meson cloud contamination (kaon cloud) is expected to be small due to the large kaon mass, the lattice QCD data may be considered to describe the physical $\Omega^{-}$.

We consider a model where the $\Omega^{-}$is described by a dominant $S$ state and two different $D$-state components: one with total quark spin-1/2, another with the total quark spin-3/2 $[45,48]$. Each radial wave function is parametrized by a unique momentum-range parameter. The number of free parameters is then five: two $D$-state mixture coefficients and three momentum-range parameters.

Our model for the $\Omega^{-}$[48] was calibrated by the $\Omega^{-}$ lattice QCD data from Ref. [64]. The free parameters of the radial wave functions and $D$-state mixture coefficients of our model were adjusted by the lattice QCD results for the form factors $G_{E 0}, G_{M 1}$, and $G_{E 2}$ for $Q^{2}<2 \mathrm{GeV}^{2}$. The model was then used to estimate the functions $G_{E 2}$ (electric qudrupole form factor) and $G_{M 3}\left(Q^{2}\right)$ (magnetic octupole form factor).

In the case of the electric quadrupole form factor $\left(G_{E 2}\right)$, one obtains a consistent description of the lattice QCD data, which allows the determination of the electric quadrupole moment from $G_{E 2}(0)=0.680 \pm 0.012$. For $G_{M 3}$, however, the lattice QCD simulations are restricted to the result for $Q^{2}=0.23 \mathrm{GeV}^{2}, G_{M 3}=1.25 \pm 7.50[48,65]$ (which has a significant error bar). From the form factors $G_{E 0}, G_{M 1}, G_{E 2}$, and $G_{M 3}$, we calculate the function $G$ based on the results in Appendix A. The results are presented in the left panel of Fig. 5.

Our estimates for the electromagnetic form factors in the timelike region of $\Omega^{-}$should be taken with caution, since the model used for the radial wave functions was not chosen in order to describe the large- $Q^{2}$ region but rather fitted to the $Q^{2}<2 \mathrm{GeV}^{2}$ data. For that reason, the falloff of $G_{E 0}$ and $G_{M 1}$ at large $Q^{2}$ is determined by the $1 / Q^{6}$ behavior and not by the falloff of PQCD $\left(1 / Q^{4}\right)$.

From Fig. 5 (left panel), we conclude that our results for $G$ overestimate the data. In order to understand this result, we examine the magnitude of the higher multipole form factors $G_{E 2}$ and $G_{M 3}$. If we drop these contributions, we obtain the results presented in the right panel of Fig. 5. In this case, we observe a close agreement with the data. From this analysis, we can conclude that the deviation from the data comes from the form factors $G_{E 2}$ and $G_{M 3}$. We have confirmed that it is the function $G_{M 3}$ that originates a contribution that makes the total results differ from the data. Our model gives $G_{M 3}(0) \simeq 15.5$. The result presented in the right panel of Fig. 5 is more compatible with $G_{M 3}(0) \approx 1$. We then conclude that the timelike data are more consistent with a small magnitude for the function $G_{M 3}$.

The value of $G_{M 3}(0)$ has been estimated based on different frameworks. Light front QCD sum rules predict $G_{M 3}(0)=64.3 \pm 16.1$ [66]. Estimates based on a noncovariant quark model give $G_{M 3}(0)=48.2$ for a symmetric $S U_{F}(3)$ quark model and $G_{M 3}(0)=12.1$ when the symmetry is broken [67]. Our estimate is then close to the lower estimate for $G_{M 3}(0)$, and it is more consistent with the estimate that breaks $S U_{F}(3)$. The timlike data, however, seem to indicate that $G_{M 3}(0)$ may be even smaller.

It is worth noting that the function $G_{M 3}$ is, at the moment, poorly estimated. On the contrary, the functions $G_{E 0}, G_{M 1}$, and $G_{E 2}$ are well determined by the lattice QCD data. The present result suggests the need for a determination of $G_{M 3}$ by a combined study of more accurate lattice QCD data with the very recent timelike region data for $G$ in the region $q^{2} \approx 16 \mathrm{GeV}^{2}$ [11]. In future studies, the expected PQCD falloff of the form factors for very large $q^{2}$ should also be taken into account. 


\section{OUTLOOK AND CONCLUSIONS}

A relativistic quark model which was successful in the description of the baryon electromagnetic form factors in the spacelike region was extended to the timelike region. Our $S U_{F}(3)$ model provides a fair description of the data both in the spacelike and timelike regions.

The extension of the model from the spacelike into the timelike regions uses asymptotic reflection symmetry relations connecting the electromagnetic elastic form factors in the two different regions. The theoretical uncertainty in our predictions for the timelike region was presented. An important conclusion is that the measured data are consistent with the asymptotic relations of Eqs. (4.1) and (4.2) originated from general principles as unitarity and analyticity. Finite corrections for $q^{2}$ still have a role in the strength of the form factors for $q^{2}=10-30 \mathrm{GeV}^{2}$, since within this range the differences between the results obtained from $G_{l}^{\mathrm{SL}}\left(-q^{2}\right), G_{l}^{\mathrm{SL}}\left(2 M_{B}^{2}-q^{2}\right)$, and $G_{l}^{\mathrm{SL}}\left(4 M_{B}^{2}-q^{2}\right)(l=M, E)$ show that the strict $q^{2} \rightarrow \infty$ limit is not yet attained numerically within that region. On the other hand, the fact that the data are within the theoretical uncertainty of our model seems to indicate that the reflection symmetry center point is inside the unphysical region $] 0,4 M_{B}^{2}$ [, where $M_{B}$ is the baryon mass.

Our model leads to the correct PQCD asymptotic power law behavior of the electromagnetic form factors. But an important conclusion of this work is that the PQCD limit onset $G \propto 1 / q^{4}$ is way above the region where the reflection symmetry relations are valid. We found that only beyond the region of $q^{2}: 30-50 \mathrm{GeV}^{2}$, the PQCD power law was observed. This was interpreted as an interplay of the two scales entering the model: the meson mass scales that determine the quark electromagnetic current and the momentum-range scales determined by the extension of the hyperons.

In the present work, our main focus was on the baryon octet since the available data are mostly on that family of baryons, and therefore, the comparison with the data enabled us to better probe our model in the timelike region. Our framework can also be applied to all baryons of the decuplet, and as an example, we presented our results for the $\Omega^{-}$baryon and compared them with the new data from CLEO.

Under study is the possible extension of the present model to charmed baryons. By this extension, the model can be applied to the $e^{+} e^{-} \rightarrow \Lambda_{c}^{+} \bar{\Lambda}_{c}^{+}$process to estimate the $\Lambda_{c}^{+}$timelike electromagnetic form factors, which were recently measured at BES-III [68].

\section{ACKNOWLEDGMENTS}

G. R. was supported by the Fundação de Amparo à Pesquisa do Estado de São Paulo (FAPESP): Project No. 2017/02684-5, Grant No. 2017/17020-BCO-JP. M. T.P. was supported in part by Fundação para a
Ciência e a Tecnologia Grant No. CFTP-FCT (UID/FIS/ 00777/2015). K. T. was supported by Conselho Nacional de Desenvolvimento Científico e Tecnológico, Processes No. 313063/2018-4 and No. 426150/2018-0, and FAPESP Process No. 2019/00763-0, and his work was also part of the projects Instituto Nacional de Ciência e TecnologiaNuclear Physics and Applications, Brazil, Process No. 464898/2014-5 and FAPESP Temático, Brazil, Process No. 2017/05660-0. M. T. P. thanks Gernot Eichmann and Alfred Stadler for useful discussions.

\section{APPENDIX A: $3 / 2^{+}$BARYONS}

As discussed in the main text, the relations (2.1) and (2.2) can be used for $3 / 2^{+}$baryons if the form factors $G_{M}$ and $G_{E}$ are expressed as combinations of electric form factors (electric charge $G_{E 0}$ and electric quadrupole $G_{E 2}$ ) and magnetic form factors (magnetic dipole $G_{M 1}$ and magnetic octupole $G_{M 3}$ ).

According to Ref. [20], we should use the following replacements:

$$
\begin{gathered}
\left|G_{E}\right|^{2} \rightarrow 2\left|G_{E 0}\right|^{2}+\frac{8}{9} \tau^{2}\left|G_{E 2}\right|^{2}, \\
\left|G_{M}\right|^{2} \rightarrow \frac{10}{9}\left|G_{M 1}\right|^{2}+\frac{32}{5} \tau^{2}\left|G_{M 3}\right|^{2},
\end{gathered}
$$

where $\tau=\frac{q^{2}}{4 M_{B}^{2}}$.

\section{APPENDIX B: DETAILS OF THE MODEL}

Below we describe some details of the model, including the spin and flavor wave functions, the explicit form of the quark form factors, the parameters of the model, and the values of the normalization factors due to the inclusion of pion cloud contributions.

\section{Baryon wave functions}

In the covariant spectator quark model, the spin states associated with Eq. (3.1) are represented by [34,39]

$$
\phi_{S}^{0}=u_{B}(P, s), \quad \phi_{S}^{1}=-\left(\varepsilon_{\lambda}^{*}\right)_{\alpha}(P) U_{B}^{\alpha}(P, s),
$$

where $u_{B}$ is the Dirac spinor of the baryon, $s$ is the baryon spin projection, $\lambda$ represents the polarization of the diquark, and

$$
U_{B}^{\alpha}(P, s)=\frac{1}{\sqrt{3}} \gamma_{5}\left(\gamma^{\mu}-\frac{P^{\alpha}}{M_{B}}\right) u_{B}(P, s) .
$$

The wave function described by Eqs. (3.1) and (B1) generalize the nonrelativistic wave function in a covariant form [39]. The flavor states $\left|M_{S}\right\rangle$ (mixed symmetric) and $\left|M_{A}\right\rangle$ (mixed antisymmetric) for all octet baryons are presented in Table IV. Although the results for the nucleon 
TABLE IV. Flavor wave functions of the octet baryons.

\begin{tabular}{lcc}
\hline \hline$B$ & $\left|M_{A}\right\rangle$ & $\left|M_{S}\right\rangle$ \\
\hline$p$ & $\frac{1}{\sqrt{2}}(u d-d u) u$ & $\frac{1}{\sqrt{6}}[(u d+d u) u-2 u u d]$ \\
$n$ & $\frac{1}{\sqrt{2}}(u d-d u) d$ & $-\frac{1}{\sqrt{6}}[(u d+d u) d-2 d d u]$ \\
$\Lambda^{0}$ & $\frac{1}{\sqrt{12}}[s(d u-u d)-(d s u-u s d)-2(d u-d u) s]$ & $\frac{1}{2}[(d s u-u s d)+s(d u-u d)]$ \\
$\Sigma^{+}$ & $\frac{1}{\sqrt{2}}(u s-s u) u$ & $\frac{1}{\sqrt{6}}[(u s+s u) u-2 u u s]$ \\
$\Sigma^{0}$ & $\frac{1}{2}[(d s u+u s d)-s(u d+d u)]$ & $\frac{1}{\sqrt{12}}[s(d u+u d)+(d s u+u s d)-2(u d+d u) s]$ \\
$\Sigma^{-}$ & $\frac{1}{\sqrt{2}}(d s-s d) d$ & $\frac{1}{\sqrt{6}}[(s d+d s) d-2 d d s]$ \\
$\Xi^{0}$ & $\frac{1}{\sqrt{2}}(u s-s u) s$ & $-\frac{1}{\sqrt{6}}[(u d+d u) s-2 s s u]$ \\
$\Xi^{-}$ & $\frac{1}{\sqrt{2}}(d s-s d) s$ & $-\frac{1}{\sqrt{6}}[(d s+s d) s-2 s s d]$ \\
\hline \hline
\end{tabular}

are not discussed in the present work, we include the proton and neutron states for completeness.

\section{Quark form factors}

To parametrize the quark current (3.6), we adopt the structure inspired by the VMD mechanism as in Refs. [35,39]:

$$
\begin{aligned}
& f_{1 \pm}=\lambda_{q}+\left(1-\lambda_{q}\right) \frac{m_{v}^{2}}{m_{v}^{2}+Q^{2}}+c_{ \pm} \frac{M_{h}^{2} Q^{2}}{\left(M_{h}^{2}+Q^{2}\right)^{2}}, \\
& f_{10}=\lambda_{q}+\left(1-\lambda_{q}\right) \frac{m_{\phi}^{2}}{m_{\phi}^{2}+Q^{2}}+c_{0} \frac{M_{h}^{2} Q^{2}}{\left(M_{h}^{2}+Q^{2}\right)^{2}}, \\
& f_{2 \pm}=\kappa_{ \pm}\left\{d_{ \pm} \frac{m_{v}^{2}}{m_{v}^{2}+Q^{2}}+\left(1-d_{ \pm}\right) \frac{M_{h}^{2}}{M_{h}^{2}+Q^{2}}\right\}, \\
& f_{20}=\kappa_{s}\left\{d_{0} \frac{m_{\phi}^{2}}{m_{\phi}^{2}+Q^{2}}+\left(1-d_{0}\right) \frac{M_{h}^{2}}{M_{h}^{2}+Q^{2}}\right\},
\end{aligned}
$$

where $m_{v}, m_{\phi}$, and $M_{h}$ are the masses, respectively, corresponding to the light vector meson $m_{v} \simeq m_{\rho} \simeq m_{\omega}$, the $\phi$ meson (associated with an $s \bar{s}$ state), and an effective heavy meson with mass $M_{h}=2 M_{N}$ to represent the shortrange phenomenology. The parameter $\lambda_{q}$ is determined by the study of deep inelastic scattering [39]. The relation between the quark anomalous magnetic moments $\kappa_{u}$ and $\kappa_{d}$ is $\kappa_{+}=2 \kappa_{u}-\kappa_{d}$ and $\kappa_{-}=\frac{1}{3}\left(2 \kappa_{u}+\kappa_{d}\right)$.

We consider the parametrization from Refs. [34,39] in the study of the nucleon and decuplet systems, except for the quark anomalous magnetic moments $\kappa_{+}$and $\kappa_{-}$. Those coefficients are readjusted in our study of the octet baryon electromagnetic form factors in order to take into account the pion cloud effects. The value of $\kappa_{s}$ is fixed by the magnetic moment of the $\Omega^{-}$.

The parametrization from Eq. (B3) for the three sectors includes the quark anomalous magnetic moments (three parameters) and six extra parameters. Since the quark anomalous magnetic moments can be fixed independently by the proton, the neutron and the $\Omega^{-}$magnetic moments, we have six parameters to adjust. We reduce the number to five using the isospin symmetry for the Pauli form factor $\left(d_{+}=d_{-}\right)$. All parameters of the quark current are included in Table V. The results in boldface indicate the values fixed by the study of the octet baryons with pion cloud dressing.

\section{Octet baryon form factors}

The valence quark contributions to the octet baryon electromagnetic form factors are then determined by the combination of the quark form factors and the parametrization of the radial wave functions according to Eqs. (3.8) and (3.9).

The parameters associated with the radial wave functions (3.2) are presented in Table VI. The coefficients $j_{i}^{A, S}$ $(i=1,2)$ depend on the quark form factors according to the results from Table VII. Again, the expressions for the nucleon are included for completeness.

\section{Pion cloud dressing}

In the low- $Q^{2}$ region, it is necessary to include the effects of the pion cloud dressing of the baryons. In the study of the electromagnetic structure of the octet baryons, those effects are taken into account in an effective way. There are two main contributions to take into account: the contributions

TABLE V. Parameters associated with the quark current. In this notation, $\kappa_{s}=\kappa_{0}$. For $\lambda_{q}$, we use $\lambda_{q}=1.22$ [39].

\begin{tabular}{cccc}
\hline \hline$a$ & $\kappa_{a}$ & $c_{a}$ & $d_{a}$ \\
\hline+ & $\mathbf{1 . 4 6 2}$ & 4.160 & -0.686 \\
- & $\mathbf{1 . 7 5 6}$ & 1.160 & -0.686 \\
0 & 1.462 & 4.427 & -1.860 \\
\hline \hline
\end{tabular}

TABLE VI. Parameters of the radial wave functions (3.2). Recall that $\beta_{1}$ is the global long-range parameter. The short-range parameters are $\beta_{2}$ (nucleon), $\beta_{3}(\Lambda$ and $\Sigma)$, and $\beta_{4}(\Xi)$.

\begin{tabular}{lccc}
\hline \hline$\beta_{1}$ & $\beta_{2}$ & $\beta_{3}$ & $\beta_{4}$ \\
\hline 0.0440 & 0.9077 & 0.7634 & 0.4993 \\
\hline \hline
\end{tabular}


TABLE VII. Mixed symmetric and antisymmetric coefficients for the octet baryons.

\begin{tabular}{lcc}
\hline \hline$B$ & $j_{i}^{S}$ & $j_{i}^{A}$ \\
\hline$p$ & $\frac{1}{6}\left(f_{i+}-f_{i-}\right)$ & $\frac{1}{6}\left(f_{i+}+3 f_{i-}\right)$ \\
$n$ & $\frac{1}{6}\left(f_{i+}+f_{i-}\right)$ & $\frac{1}{6}\left(f_{i+}-3 f_{i-}\right)$ \\
$\Lambda^{0}$ & $\frac{1}{6} f_{i+}$ & $\frac{1}{18}\left(f_{i+}-4 f_{i 0}\right)$ \\
$\Sigma^{+}$ & $\frac{1}{18}\left(f_{i+}+3 f_{i-}-4 f_{i 0}\right)$ & $\frac{1}{6}\left(f_{i+}+3 f_{i-}\right)$ \\
$\Sigma^{0}$ & $\frac{1}{36}\left(2 f_{i+}-8 f_{i 0}\right)$ & $\frac{1}{6} f_{i+}$ \\
$\Sigma^{-}$ & $\frac{1}{18}\left(f_{i+}-3 f_{i-}-4 f_{i 0}\right)$ & $\frac{1}{6}\left(f_{i+}-3 f_{i-}\right)$ \\
$\Xi^{0}$ & $\frac{1}{18}\left(2 f_{i+}+6 f_{i-}-2 f_{i 0}\right)$ & $-\frac{1}{3} f_{i 0}$ \\
$\Xi^{-}$ & $\frac{1}{18}\left(2 f_{i+}-6 f_{i-}-2 f_{i 0}\right)$ & $-\frac{1}{3} f_{i 0}$ \\
\hline \hline
\end{tabular}

associated with the photon coupling with the pion and the contributions associated with the photon coupling with intermediate octet baryon states. All these processes can be parametrized based on an $S U(3)$ model for the pion-baryon interaction using five independent couplings and two cutoffs (regulate falloff of pion cloud contributions) [34].

The main consequence of the inclusion of the pion cloud contributions is that the estimates of $G_{E B}^{B}$ and $G_{M B}^{B}$ from the valence quark contributions to the octet baryon form factors are modified by the normalization of the wave function which combine valence and pion cloud contributions $\left(\delta G_{E B}\right.$ and $\left.\delta G_{M B}\right)$ :

$$
\begin{gathered}
G_{E B}=Z_{B}\left[G_{E B}^{B}+\delta G_{E B}\right], \\
G_{M B}=Z_{B}\left[G_{M B}^{B}+\delta G_{M B}\right] .
\end{gathered}
$$

The explicit expressions for $\delta G_{E B}$ and $\delta G_{M B}$ can be found in Refs. $[34,42]$. When we increase $Q^{2}$, the pion cloud contributions are strongly suppressed since they are regulated by higher order multipoles with square cutoffs of the order 0.8 and $1.2 \mathrm{GeV}^{2}$ [34].
TABLE VIII. Coefficients $a_{B}$ associated with the normalization of the octet baryon wave functions, and numerical result for the normalization constant $Z_{B}$. The $S U(6)$ value is $\alpha=0.6$.

\begin{tabular}{lcc}
\hline \hline$B$ & $a_{B}$ & $Z_{B}$ \\
\hline$N$ & 1 & 0.885 \\
$\Lambda$ & $\frac{4}{3} \alpha^{2}$ & 0.941 \\
$\Sigma$ & $\frac{1}{3}\left(8-16 \alpha+10 \alpha^{2}\right)$ & 0.929 \\
$\Xi$ & $(1-2 \alpha)^{2}$ & 0.995 \\
\hline \hline
\end{tabular}

The parameters associated with the valence quark contributions are determined by fits to the lattice QCD results for the octet baryon electromagnetic form factors. The parameters associated with the pion cloud contribution are fixed by the physical data (nucleon electromagnetic form factors and octet baryon magnetic moments).

In Eqs. (B4) and (B5), the factor $Z_{B}$ can be written as $[34,42,52]$

$$
Z_{B}=\frac{1}{1+3 a_{B} B_{1}},
$$

where $a_{B}$ is a coefficient determined by the $S U(3)$ symmetry, and $B_{1}$ is a parameter which determines the nucleon normalization $\left(Z_{N}\right)$ based on the normalization $a_{N}=1$.

The normalization constant for the nucleon $Z_{N}=$ $1 /\left(1+3 B_{1}\right)$, meaning that the contribution from the valence quarks for the proton charge is $Z_{N}$ and the contribution from the pion cloud $3 B_{1} Z_{N}$. One concludes then that the relative pion cloud contribution to the proton electric form factor is $3 B_{1}$, which implies that the normalization $Z_{N}$ can be determined by the estimate of the pion cloud contribution based on the comparison between the valence quark contributions and the data, and vice versa $B_{1}=\frac{1}{3} \frac{1-Z_{N}}{Z_{N}}$. The values of $Z_{B}$ based on the value for $B_{1}$ and $a_{B}$ are presented in Table VIII.
[1] I. G. Aznauryan et al., Int. J. Mod. Phys. E 22, 1330015 (2013).

[2] C. F. Perdrisat, V. Punjabi, and M. Vanderhaeghen, Prog. Part. Nucl. Phys. 59, 694 (2007).

[3] A. Denig and G. Salme, Prog. Part. Nucl. Phys. 68, 113 (2013).

[4] S. Pacetti, R. B. Ferroli, and E. Tomasi-Gustafsson, Phys. Rep. 550-551, 1 (2015).

[5] N. Cabibbo and R. Gatto, Phys. Rev. 124, 1577 (1961).

[6] P. Kroll, T. Pilsner, M. Schurmann, and W. Schweiger, Phys. Lett. B 316, 546 (1993).

[7] R. Jakob, P. Kroll, M. Schurmann, and W. Schweiger, Z. Phys. A 347, 109 (1993).
[8] R. L. Jaffe and F. Wilczek, Phys. Rev. Lett. 91, 232003 (2003).

[9] F. Wilczek, in From Fields to Strings: Circumnavigating Theoretical Physics, edited by M. Shifman et al., Vol. 1 (World Scientific, Singapore, 2005), pp. 77-93, https:// doi.org/10.1142/9789812775344_0007.

[10] A. Selem and F. Wilczek, in New Trends in HERA Physics (World Scientific, Singapore, 2006), https://doi.org/ 10.1142/9789812773524_0030.

[11] S. Dobbs, K. K. Seth, A. Tomaradze, T. Xiao, and G. Bonvicini, Phys. Rev. D 96, 092004 (2017).

[12] S. Dobbs, A. Tomaradze, T. Xiao, K. K. Seth, and G. Bonvicini, Phys. Lett. B 739, 90 (2014). 
[13] B. Aubert et al. (BABAR Collaboration), Phys. Rev. D 73, 012005 (2006).

[14] K. K. Seth, S. Dobbs, Z. Metreveli, A. Tomaradze, T. Xiao, and G. Bonvicini, Phys. Rev. Lett. 110, 022002 (2013).

[15] K. Schönning and C. Li, EPJ Web Conf. 137, 12002 (2017).

[16] C. Li (BESIII Collaboration), J. Phys. 742, 012020 (2016).

[17] B. Aubert et al.(BABAR Collaboration), Phys. Rev. D 76, 092006 (2007).

[18] M. Ablikim et al. (BESIII Collaboration), Phys. Rev. D 87, 112011 (2013).

[19] M. Ablikim et al. (BESIII Collaboration), Phys. Rev. D 97, 032013 (2018).

[20] J. G. Korner and M. Kuroda, Phys. Rev. D 16, 2165 (1977).

[21] J. Haidenbauer, T. Hippchen, K. Holinde, B. Holzenkamp, V. Mull, and J. Speth, Phys. Rev. C 45, 931 (1992).

[22] A. Z. Dubnickova, S. Dubnicka, and P. Strizenec, Czech. J. Phys. 43, 1177 (1993).

[23] O. D. Dalkarov, P. A. Khakhulin, and A. Y. Voronin, Nucl. Phys. A833, 104 (2010).

[24] J. Haidenbauer and U. G. Meissner, Phys. Lett. B 761, 456 (2016).

[25] Y. Yang and Z. Lu, Mod. Phys. Lett. A 33, 1850133 (2018).

[26] X. Cao, J. P. Dai, and Y. P. Xie, Phys. Rev. D 98, 094006 (2018).

[27] Y. Yang, D. Y. Chen, and Z. Lu, Phys. Rev. D 100, 073007 (2019).

[28] B. Singh et al. (PANDA Collaboration), Phys. Rev. D 95, 032003 (2017).

[29] S. J. Brodsky and G. R. Farrar, Phys. Rev. Lett. 31, 1153 (1973).

[30] S. J. Brodsky and G. R. Farrar, Phys. Rev. D 11, 1309 (1975).

[31] G. P. Lepage and S. J. Brodsky, Phys. Rev. D 22, 2157 (1980).

[32] C. E. Carlson and F. Gross, Phys. Rev. D 36, 2060 (1987).

[33] A. V. Belitsky, X. d. Ji, and F. Yuan, Phys. Rev. Lett. 91, 092003 (2003).

[34] G. Ramalho and K. Tsushima, Phys. Rev. D 87, 093011 (2013).

[35] G. Ramalho, K. Tsushima, and F. Gross, Phys. Rev. D 80, 033004 (2009).

[36] G. Eichmann, P. Duarte, M. T. Peña, and A. Stadler, Phys. Rev. D 100, 094001 (2019).

[37] J. Haidenbauer, X.-W. Kang, and U.-G. Meissner, Nucl. Phys. A929, 102 (2014).

[38] C. Tzara, Nucl. Phys. B18, 246 (1970).

[39] F. Gross, G. Ramalho, and M. T. Peña, Phys. Rev. C 77, 015202 (2008).

[40] F. Gross, G. Ramalho, and M. T. Peña, Phys. Rev. D 85, 093005 (2012).

[41] G. Ramalho, Few-Body Syst. 59, 92 (2018).

[42] G. Ramalho, K. Tsushima, and A. W. Thomas, J. Phys. G 40, 015102 (2013).
[43] G. Ramalho, J. P. B. C. De Melo, and K. Tsushima, Phys. Rev. D 100, 014030 (2019).

[44] G. Ramalho and K. Tsushima, Phys. Rev. D 86, 114030 (2012).

[45] G. Ramalho, M. T. Peña, and F. Gross, Phys. Rev. D 78, 114017 (2008).

[46] G. Ramalho and M. T. Peña, Phys. Rev. D 80, 013008 (2009).

[47] G. Ramalho, M. T. Peña, J. Weil, H. van Hees, and U. Mosel, Phys. Rev. D 93, 033004 (2016).

[48] G. Ramalho and M. T. Peña, Phys. Rev. D 83, 054011 (2011).

[49] G. Ramalho and M. T. Peña, J. Phys. G 36, 115011 (2009).

[50] G. Ramalho, M. T. Peña, and F. Gross, Eur. Phys. J. A 36, 329 (2008).

[51] G. Ramalho and M. T. Peña, J. Phys. G 36, 085004 (2009); G. Ramalho, M. T. Peña, and F. Gross, Phys. Rev. D 81, 113011 (2010).

[52] F. Gross, G. Ramalho, and K. Tsushima, Phys. Lett. B 690, 183 (2010).

[53] G. Ramalho, D. Jido, and K. Tsushima, Phys. Rev. D 85, 093014 (2012).

[54] G. Ramalho, Phys. Rev. D 94, 114001 (2016); G. Ramalho, Eur. Phys. J. A 54, 75 (2018).

[55] T. K. Pedlar et al. (CLEO Collaboration), Phys. Rev. Lett. 95, 261803 (2005).

[56] T. A. Armstrong et al. (E760 Collaboration), Phys. Rev. Lett. 70, 1212 (1993).

[57] M. Ambrogiani et al. (E835 Collaboration), Phys. Rev. D 60, 032002 (1999).

[58] E. A. Kuraev, E. Tomasi-Gustafsson, and A. Dbeyssi, Phys. Lett. B 712, 240 (2012).

[59] A. J. R. Puckett et al., Phys. Rev. C 96, 055203 (2017); 98, 019907(E) (2018).

[60] C. Alexandrou, G. Koutsou, J. W. Negele, and A. Tsapalis, Phys. Rev. D 74, 034508 (2006).

[61] D. Bisello et al. (DM2 Collaboration), Z. Phys. C 48, 23 (1990).

[62] A. Antonelli et al., Nucl. Phys. B517, 3 (1998).

[63] M. N. Achasov et al., Phys. Rev. D 90, 112007 (2014).

[64] C. Alexandrou, T. Korzec, G. Koutsou, J. W. Negele, and Y. Proestos, Phys. Rev. D 82, 034504 (2010).

[65] S. Boinepalli, D. B. Leinweber, P. J. Moran, A. G. Williams, J. M. Zanotti, and J. B. Zhang, Phys. Rev. D 80, 054505 (2009).

[66] T. M. Aliev, K. Azizi, and M. Savci, Phys. Lett. B 681, 240 (2009).

[67] A. J. Buchmann and E. M. Henley, Eur. Phys. J. A 35, 267 (2008).

[68] M. Ablikim et al. (BESIII Collaboration), Phys. Rev. Lett. 120, 132001 (2018). 